\title{
Reconcile Discrepancies of Current Syngas Kinetics Models by Considering Turbulence Effects on Ignition Delay at Gas-Turbine Relevant Operating Conditions
}

\author{
Matthias Ihme* \\ Department of Aerospace Engineering, University of Michigan \\ 1320 Beal Avenue, 3017 FXB Building, Ann Arbor, MI 48109-2140
}

\begin{abstract}
With the increasing interest in utilizing syngas in gas turbine applications, the characterization of $\mathrm{H}_{2} / \mathrm{CO}$ reaction chemistry under high-pressure and moderate-temperature operating conditions has been the focus of recent investigations. Different chemical-kinetics and hydrodynamic processes have been identified as being responsible for the discrepancies between experimental measurements and kinetic predictions of syngas ignition delay times. This paper complements previous studies, and provides insight on the role of turbulence fluctuations and small-scale mixture inhomogeneities on the syngas combustion process. To this end, an analytic model has been developed that describes the turbulence amplification and combustion in the so-called adiabatic core region of a rapid compression machine (RCM). In this formulation, enhancement of turbulence and small-scale mixture fluctuations during the RCM compression phase are modeled using rapid distortion theory. The subsequent ignition and combustion processes are described using a Lagrangian Fokker-Planck model which considers turbulence/chemistry interaction and detailed reaction chemistry. The model was applied to different syngas mixtures and operating conditions, including pressures up to 20 atm and temperatures between 600 and $1300 \mathrm{~K}$. Parametric studies showed that the model captures experimentally observed trends of reduced ignition delay and prolonged reaction progress during the ignition phase. A Damköhler criterion was introduced in order to characterize the sensitivity of the induction chemistry to turbulence fluctuations. Results suggest that syngas mixtures with Damhöhler numbers below 100 exhibit increasing sensitivity to turbulence and mixture fluctuations. Initial turbulence levels of less than 0.01 percent of the mean flow are sufficient in reducing the ignition delay times by several orders of magnitude. This study shows that the turbulence/chemistry interaction plays an equally important role in affecting the syngas ignition chemistry, and requires consideration in addition to chemical-kinetics and hydrodynamic processes, previously identified as leading mechanisms for the observed discrepancy in syngas combustion at low-temperature operating conditions.
\end{abstract}

\section{Introduction}

Accompanied with the rapid economic growth in the developing nations of Asia is an increasing energy demand, which, according to the International Energy Outlook $2009,{ }^{1}$ is predicted to exceed the U.S. energy consumption by more than 65 percent by 2030. In the presence of the finite fossil energy reserves and growing environmental concerns, the utilization of reformed fuels provides an attractive alternative for accommodating the increasing energy demand. Among these reformed fuels, syngas has been identified as attractive solution for power generation applications. Specifically, in coal-based integrated combined-cycle (IGCC) power generation application, syngas, which contains hydrogen $\left(\mathrm{H}_{2}\right)$ and carbon monoxide $(\mathrm{CO})$ as primary fuel components, is generated through a coal gasification process. ${ }^{2}$ After sulfur oxides, particulates and other pollutants are removed, the syngas is combusted in a gas turbine cycle, and excess heat is converted into a subsequent steam turbine cycle.

*Assistant Professor, Member AIAA, (mihme@umich.edu) 
Despite the enormous opportunities in improving combustion efficiency and reducing pollutant emissions, syngas combustion introduces significant technological and scientific challenges. These issues mainly arise from process-related variations in the syngas composition, high hydrogen concentrations, and overall lean operating conditions. In addition, IGCC gas turbines are operated under high pressure (up to 30 bar) and intermediate temperature $(T<1000 \mathrm{~K})$ conditions, and the syngas combustion under these conditions is currently only insufficiently understood.

The renewed interest in syngas combustion led to numerous experimental studies in order to obtain improved understanding about the combustion-physical properties and reaction chemistry at gas-turbine relevant operating conditions. These experiments are typically conducted in shock tubes, flow reactors, and rapid compression machines (RCMs), and Refs. [3-6] provide comprehensive overviews about experimental investigations and measurements for ignition delay time, flame speed, and other combustion-physical properties.

Interestingly, comparisons of ignition delay times from measurements and computations exhibit significant discrepancies ${ }^{6}$ that increase with decreasing initial temperature of the syngas/air mixture. To reconcile these discrepancies, different explanations have been suggested, and updates on the $\mathrm{H}_{2} / \mathrm{CO}$ kinetic models have been proposed. ${ }^{3,7,8}$ In particular, gas impurities, surface-catalytic effects, wall-heat transfer, and large-scale mixing effects have been pointed out as potential sources. Although uncertainties for rate constants of reactions involving hydroperoxyl radicals $\left(\mathrm{HO}_{2}\right)$ and hydrogen peroxide $\left(\mathrm{H}_{2} \mathrm{O}_{2}\right)$ have been recognized, it was suggested that their contributions only partially explain the observed discrepancies in the ignition delay.

Over recent years, computational and experimental studies have been conducted to characterize the flow field structure and wall heat transfer in RCMs. ${ }^{9-12}$ Intrusive and non-intrusive measurements of temperature and species have been performed to demonstrate the existence of a so-called adiabatic core region. In this adiabatic core region, that typically extends up to $70 \%$ across the diameter of the test section, the gas mixture is not affected by wall heat losses and boundary layer effects. The mixture contained in this volume is isentropically compressed, and the combustion of the nominally uniform composition can be approximated as homogeneous reactor system. In this context it is noted that the existence of this adiabatic core region is fundamental in order to relate the rapid compression experiments to zero-dimensional homogeneous ignition studies. Computational studies led to important modifications of the piston-crown design in order to suppress the generation of piston corner vortices, which are known to entrain cold fluid from the boundary layer, and can destroy the adiabatic core region.

While many, if not all, of these investigations focused on the large-scale fluid motion and wall heat transfer effects, the role of the turbulence on the ignition and combustion in RCMs has so far not been appreciated. The objective of this contribution is to address this aspect. To this end, a model will be developed that enables the quantitative characterization of the turbulence fluctuation and small-scale variations in the mixture composition during the compression and ignition of the gas mixture.

The potential importance of the turbulence in a RCM can be assessed from the Reynolds number Re, which can be estimated as

$$
\operatorname{Re}=\frac{l_{1}^{*}}{\tau^{*}} \frac{d^{*}}{\nu^{*}}
$$

where the ratio $l_{1}^{*} / \tau^{*}$ is the characteristic speed of the piston, $l_{1}^{*}$ is the length of the driven section, $\tau^{*}$ is the compression time, $d^{*}$ is the diameter of the test section, and $\nu^{*}$ is the kinematic viscosity of the gas mixture. Typical Reynolds numbers for RCMs are in the range $\operatorname{Re} \approx \mathcal{O}\left(10^{4}-10^{5}\right)$, suggesting that the compression and subsequent ignition is not unaffected by turbulence. This argument is supported through recent measurements by Guibert et al. ${ }^{13}$ in which they investigated the influence of post-compression turbulence levels on the combustion process.

In a RCM, different sources of turbulence can be identified. The first source arises from the filling process. Specifically, the filling process of the driven section with the fresh test gas mixture is accompanied with the generation of small-scale turbulence fluctuations that are approximately homogeneously distributed in the entire test section. If the compression phase is initiated directly after the filling process, providing insufficient time for the complete decay, the initially introduced turbulence is amplified during the compression. Similarly, the incomplete mixing of the test mixture can lead to fluctuations and potential large-scale stratifications of the test gas composition. While these contributions can be minimized through the external preparation of the test gas mixture, they may become relevant if an internal mixture preparation is utilized, or when reactants with different molecular weights are mixed so that preferential diffusive effects become relevant. The second mechanism arises from the wall-generated turbulence. During the compression phase, 
turbulence is produced in the boundary layer, which is entrained into the core region by convective and diffusive transport, and further amplified through the mean strain interaction.

While both mechanisms can become of equal importance, this work addresses effects of the initial turbulence on the ignition and combustion of the test gas mixture as it is amplified during the compression phase. As such, the results of this analysis can be considered as conservative estimates, and it is expected that other contributions may lead to further turbulence amplifications.

To quantify the effects of turbulence on the ignition dynamics and ignition delay time, a theoretical model will be developed that consists of three components: (i) the description of the piston motion, (ii) the amplification of the initial turbulence during the compression phase, and (iii) the autoignition of the test gas mixture under consideration of the turbulence/chemistry interaction. The ignition process is described using detailed chemical mechanisms for the syngas combustion, which allows the assessment of potential discrepancies of existing reaction mechanisms.

Amplifications of turbulence and fluctuations in mixture composition are described using rapid distortion theory (RDT). RDT describes the evolution of initially homogeneous turbulence when it is subjected to rapid strain. ${ }^{14-16}$ This theory has been applied to a wide range of problems, including the compression in a piston engine by Hunt ${ }^{14}$ using the Cauchy form of the vorticity equation. In the present work, a more general RDT formulation will be presented that also enables us to consider effects of equivalence ratio stratification on the amplification of the compositional fluctuations.

The ignition of the test gas mixture is modeled by considering individual ignition kernels that are homogeneously distributed in the test section. These ignition kernels interact among each other through turbulent mixing and diffusion. To this end, a Lagrangian Fokker-Planck (LFP) model is derived, and closure is obtained using a $k-\varepsilon$ formulation and "interaction by exchange with the mean (IEM)" mixing model. The particular advantage of this model is that this LFP formulation reduces to the well-known homogeneous reactor model in the absence of turbulence and mixture inhomogeneities. In this context it is noted that effects of the flame propagation are not considered in the present model. Front-like combustion modes have been reported for hydrocarbon and hydrogen fuel mixtures. ${ }^{5,13,17}$ Images suggest that these flame-propagation modes and combustion non-uniformities are initiated by mixture impurities. ${ }^{17}$ In principle, such effects can be incorporated into the proposed model, if the underlying combustion-physical mechanisms, triggering this combustion mode, are understood.

The remainder of this paper is organized as follows. The mathematical model is developed in the next section. The experimental configuration and model parameters are summarized in Sec. III. Results are presented in Sec. IV, and the paper finishes with conclusions.

\section{Mathematical Model}

In the following, a mathematical model is developed to describe the compression and subsequent combustion process in a rapid compression machine. In this analysis, the adiabatic core region is considered. This core region is characterized by an adiabatic state, in which the gas mixture is not affected by wall heat losses, large-scale mixing, and boundary layer perturbations over the duration of the RCM operation. With this, the RCM core region is considered as a quasi one-dimensional system.

The schematic of the RCM is illustrated in Fig. 1, consisting of a driver section and a driven section, which are separated by a freely moving piston with mass $m^{*}$. A coordinate system $x_{1}^{*}$ is introduced, and the location of the piston with respect to the coordinate origin is denoted by $x_{\mathrm{P}}^{*}$. In the following, conditions in the driven and driver sections are denoted by subscripts " 1 " and "2," respectively, and all dimensional quantities are indicated by an asterisk.

To describe the kinematics of the RCM during the compression phase, the following references quantities are introduced:

$$
\begin{aligned}
\text { Length scale: } & l_{1}^{*} & =x_{\mathrm{P}}^{*}\left(t^{*}=0\right), \\
\text { Time scale: } & \tau^{*} & =\sqrt{\frac{m^{*} l_{1}^{*}}{\Delta p^{*} A^{*}}}, \\
\text { Pressure difference: } & \Delta p^{*} & =p_{2}^{*}\left(t^{*}=0\right)-p_{1}^{*}\left(t^{*}=0\right),
\end{aligned}
$$

where $p^{*}$ is the pressure and $A^{*}$ is the constant piston area. With these references properties, the following 


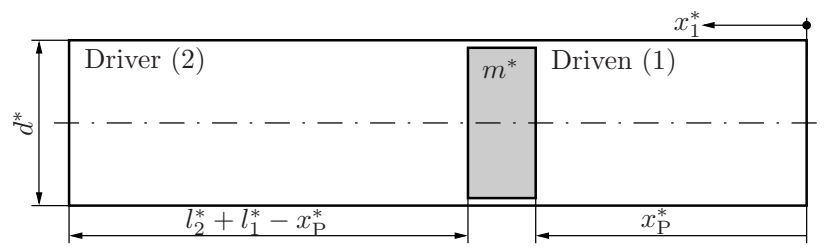

Figure 1. Schematic of a rapid compression machine. During the compression phase, the piston, shown in gray, compresses the gas mixture that is contained in the driven section. The location of the piston with respect to the origin of the coordinate systems is denoted by $x_{\mathrm{P}}^{*}$. The mass of the piston is $m^{*}$, and the initial length of the driver section is $l_{2}^{*}$. The diameter of the RCM is constant, and is denoted by $d^{*}$.

non-dimensional quantities can be defined:

$$
\begin{array}{ll}
t=\frac{t^{*}-t_{\mathrm{C}}^{*}}{\tau^{*}}, & x_{\mathrm{P}}=\frac{x_{\mathrm{P}}^{*}}{l_{1}^{*}}, \quad \ell=\frac{l_{2}^{*}}{l_{1}^{*}} \\
c=\frac{\tau^{*} c^{*}}{m^{*}}, & p=\frac{p^{*}}{\Delta p^{*}},
\end{array}
$$

where $c^{*}$ is the friction coefficient, $t^{*}$ is the time, and $t_{\mathrm{C}}^{*}$ corresponds to the duration of the compression phase, which can be evaluated from

$$
x_{\mathrm{P}}(0)=\int_{-t_{\mathrm{C}}}^{0} \dot{x}_{\mathrm{P}}\left(t^{\prime}\right) d t^{\prime}+1
$$

In this equation, $\dot{x}_{\mathrm{P}}$ is the piston speed and $t_{\mathrm{C}}=t_{\mathrm{C}}^{*} / \tau^{*}$.

The mathematical model consists of three components, describing (i) the piston motion, (ii) the compression of the gas mixture and turbulence amplification, and (iii) the subsequent ignition and combustion of the compressed fuel/air mixture. These individual modeling components are discussed in the following sections.

\section{A. Piston Motion}

The motion of the piston can be described by a second-order ordinary differential equation for a mass-springdamping system, which is derived by applying a force balance on the freely moving piston:

$$
\ddot{x}_{\mathrm{P}}+p_{2, \mathrm{~s}}\left(\frac{\ell+1-x_{\mathrm{P}}}{\ell}\right)^{-\gamma}-p_{1, \mathrm{~s}} x_{\mathrm{P}}^{-\gamma}+c \dot{x}_{\mathrm{P}}=0 .
$$

The subscript "s" denotes the initial state at the beginning of the compression phase, and the third term on the left-hand-side of Eq. (3) corresponds to the friction force. The pressure evolution in the driver and driven sections are derived from the isentropic state relation, in which the ratio of specific heats $\gamma$ is assumed to be constant during the compression phase, which is accurate within $\pm 10 \%$ for the species compositions and conditions considered here.

\section{B. Flow Field Evolution during Compression Phase}

The flow field in the driven section is characterized by the conservation equations for mass, momentum, energy and species, together with a state relation, relating pressure and density to the temperature. Using the references quantities $l_{1}^{*}, \tau^{*}, \Delta p^{*}, m^{*}$, and $A^{*}$ the governing equations can be written in non-dimensional 
form as

$$
\begin{aligned}
\mathcal{D}_{t} \rho= & -\rho \nabla \cdot \boldsymbol{u} \\
\rho \mathcal{D}_{t} \boldsymbol{u}= & -\nabla p+\frac{1}{\operatorname{Re}} \nabla \cdot \underline{\underline{\sigma}}, \\
\rho \mathcal{D}_{t} \boldsymbol{\psi}= & \frac{1}{\operatorname{ReSc}} \nabla \cdot(\rho v \nabla \boldsymbol{\psi})+\rho \dot{\boldsymbol{\omega}}_{\psi} \quad \text { with } \boldsymbol{\psi}=\{Z, C\}, \\
\rho c_{p} \mathcal{D}_{t} T= & \operatorname{Ec} \mathcal{D}_{t} p+\frac{\mathrm{Le}}{\operatorname{ReSc}} \nabla \cdot(\lambda \nabla T)+\frac{1}{\operatorname{ReSc}}\left(\rho v \sum_{i} c_{p, i} \nabla Y_{i}\right) \nabla T+ \\
& \frac{\mathrm{Ec}}{\operatorname{Re}} \underline{\underline{\sigma}}: \nabla \boldsymbol{u}+\rho \dot{\omega}_{T}, \\
p= & \frac{1}{\mathrm{Ec}} \rho R T,
\end{aligned}
$$

where $\rho$ is the density, $\boldsymbol{u}$ is the velocity vector, $\underline{\underline{\sigma}}$ is the viscous stress tensor, $Z$ is the mixture fraction, $C$ is a reaction progress variable, $v$ is the diffusivity, and $\mathcal{D}_{t}=\partial_{t}+\boldsymbol{u} \cdot \nabla$ is the substantial derivative. The Reynolds number Re is defined in Eq. (1), the Schmidt number is $\mathrm{Sc}=\nu^{*} / v^{*}$, the Lewis number is Le $=\lambda^{*} /\left(\rho^{*} c_{p}^{*} v^{*}\right)$, and $\mathrm{Ec}=\left(l_{1}^{*} / \tau^{*}\right)^{2}\left(c_{p}^{*} T^{*}\right)^{-1}$ is the Eckert number.

\section{Mean Flow}

The velocity and mixture fraction are decomposed into a mean and a fluctuating quantity following the ensemble averaging procedure, viz. $\phi=\bar{\phi}+\phi^{\prime}$. Since the Mach number is small, fluctuations in density can be neglected. It is assumed that the thermodynamic properties are not affected by variations in the composition and are therefore spatially homogeneous. Furthermore, all viscous-diffusive properties are assumed to be constant during the compression phase. With this, the mean velocity is evaluated from the mean continuity equation and piston motion as

$$
\bar{u}_{1}=\alpha(t) x_{1} \quad \text { with } \quad \alpha(t)=\frac{\dot{x}_{\mathrm{P}}(t)}{x_{\mathrm{P}}(t)},
$$

and $\alpha$ is the mean strain rate. The evolution of the mean mixture fraction and mean progress variable during the compression phase can be derived from Eqs. (4c) and (5):

$$
\overline{\boldsymbol{\psi}}=\langle\boldsymbol{\psi}\rangle+\boldsymbol{\beta}(t)\left(x_{1}-\frac{x_{\mathrm{P}}(t)}{2}\right) \quad \text { with } \quad \boldsymbol{\beta}(t)=\boldsymbol{\beta}_{\mathrm{s}} \xi(t)
$$

where $\langle\cdot\rangle$ corresponds to a volume-averaged quantity, $\boldsymbol{\beta}_{\mathrm{s}}$ denotes the stratification of mixture fraction and progress variable at the beginning of the compression, and the compression rate $\xi(t)$ is evaluated as: ${ }^{16}$

$$
\xi(t)=\exp \left\{-\int_{-t_{\mathrm{C}}}^{t} \alpha\left(t^{\prime}\right) \mathrm{d} t^{\prime}\right\}=\frac{1}{x_{\mathrm{P}}(t)} .
$$

The information about the mean flow (Eqs. (6) and (7)) is used to evaluate the amplification of the turbulence and mixture fraction fluctuations during the compression phase. For this, the rapid distortion theory (RDT) is used, and the derivation is discussed in the next section.

\section{Turbulent Flow Field}

The main objective of this contribution is to quantify how the turbulence, which is amplified during the compression, affects the ignition and combustion process in the RCM.

Rapid distortion theory lends itself to the characterization of an initially homogeneous turbulence when it is subjected to rapid strain. ${ }^{14-16}$ Specifically, RDT assumes that the evolution of the turbulence is controlled by the mean flow, and only weakly interacts with itself during the time over which the mean strain is applied. This can be characterized by the criterion

$$
\frac{1}{\alpha} \frac{u_{\mathrm{s}}^{\prime}}{l_{m}} \ll 1
$$


where $\alpha$ is the characteristic mean strain rate and $l_{m} / u_{\mathrm{s}}^{\prime}$ is the eddy life-time. Under these conditions, the non-linear terms in the fluctuating conservation equations can be neglected, and the resulting linearized equations can be written in index notation as

$$
\begin{aligned}
\partial_{i} u_{i}^{\prime} & =0 \\
\partial_{t} u_{i}^{\prime}+\alpha x_{1} \partial_{1} u_{i}^{\prime}+\alpha u_{1}^{\prime} \delta_{1 i} & =-\frac{1}{\bar{\rho}} \partial_{i} p^{\prime}, \\
\partial_{t} \boldsymbol{\psi}^{\prime}+\alpha x_{1} \partial_{1} \psi^{\prime}+u_{1}^{\prime} \boldsymbol{\beta} & =0 \\
\partial_{t} T^{\prime}+\alpha x_{1} \partial_{1} T^{\prime} & =-(\gamma-1) \alpha T^{\prime},
\end{aligned}
$$

in which the relation $p^{\prime}=\bar{\rho} R T^{\prime}$ was used to derived Eq. (9d). In Eqs. (9) all viscous-diffusive contributions are neglected as they will only add an exponentially decaying coefficient that is small compared to the straininduced amplification rate. Equations (9) can be solved in Fourier space, by substituting the Fourier mode

$$
\phi^{\prime}=\widehat{\phi}(t) \exp \{-\mathrm{i} \varkappa(t) \cdot \boldsymbol{x}\}
$$

where $\boldsymbol{\varkappa}(t)$ is the time-dependent wave-number vector. The resulting set of equations for the Fourier coefficients can be written as

$$
\begin{aligned}
d_{t} \widehat{u}_{i} & =\widehat{u}_{1} \alpha\left(\frac{2 \varkappa_{i} \varkappa_{1}}{\varkappa^{2}}-\delta_{1 i}\right), \\
d_{t} \widehat{\boldsymbol{\phi}} & =-\widehat{u}_{1} \boldsymbol{\beta}_{\mathrm{s}} \xi, \\
d_{t} \varkappa_{i} & =-\varkappa_{1} \alpha \delta_{1 i} .
\end{aligned}
$$

These equations can be solved for $\widehat{\boldsymbol{u}}, \widehat{\boldsymbol{\psi}}$, and $\varkappa$ (no summation over Greek indices) as:

$$
\begin{aligned}
\widehat{u}_{1}(t) & =\widehat{u}_{1, \mathrm{~s}} \xi \frac{\kappa^{2}}{\varkappa^{2}}, \\
\widehat{u}_{\alpha}(t) & =\widehat{u}_{1, \mathrm{~s}} \frac{\kappa_{\alpha}}{\kappa_{1}}\left(\frac{\kappa^{2}}{\varkappa^{2}}-1\right)+\widehat{u}_{\alpha, \mathrm{s}} \text { for } \alpha=\{2,3\}, \\
\widehat{\boldsymbol{\psi}}(t) & =-\widehat{u}_{1, \mathrm{~s}} \boldsymbol{\beta}_{\mathrm{s}} J+\widehat{\boldsymbol{\psi}}_{\mathrm{s}} \text { with } J=\int_{-t_{\mathrm{C}}}^{0} \xi^{2} \frac{\kappa^{2}}{\varkappa^{2}} d t^{\prime}, \\
\widehat{T}(t) & =\widehat{T}_{\mathrm{s}} \xi^{\gamma-1},
\end{aligned}
$$

where $\varkappa=\left(\kappa_{1} \xi, \kappa_{2}, \kappa_{3}\right)^{T}$, and $\boldsymbol{\kappa}$ is the initial wave number vector at the beginning of the compression phase, $t=-t_{\mathrm{C}}$.

The temporal evolution of the normal stresses and fluctuations of the mixture composition and temperature can be obtained by multiplying Eqs. (12) by its complex conjugate, substituting the isotropic energy and scalar spectra, and integrating in spherical coordinates over all wave numbers:

$$
\begin{aligned}
\frac{\left\langle u_{1}^{\prime 2}\right\rangle}{\mathcal{T}_{u}^{2}}= & \frac{3}{8 \pi} \xi^{2} \iint \frac{e_{2}^{2}+e_{3}^{2}}{\left(e_{1}^{2} \xi^{2}+e_{2}^{2}+e_{3}^{2}\right)^{2}} \sin \theta d \theta d \phi \\
\frac{\left\langle u_{\alpha}^{\prime 2}\right\rangle}{\mathcal{T}_{u}^{2}}= & \frac{3}{8 \pi} \iint\left\{\frac{e_{\alpha}^{2} e_{1}^{2}\left(1-\xi^{2}\right)}{\left(e_{1}^{2} \xi^{2}+e_{2}^{2}+e_{3}^{2}\right)^{2}} \times\right. \\
& {\left.\left[\frac{\left(1-\xi^{2}\right)\left(1-e_{1}^{2}\right)}{e_{1}^{2} \xi^{2}+e_{2}^{2}+e_{3}^{2}}-2\right]+\left(1-e_{\alpha}^{2}\right)\right\} \sin \theta d \theta d \phi, \quad \text { for } \alpha=\{2,3\} } \\
\frac{\left\langle\boldsymbol{\psi}^{\prime 2}\right\rangle}{\mathcal{T}_{\psi}^{2}}= & \frac{3}{8 \pi} \frac{\mathcal{T}_{u}^{2}}{\mathcal{T}_{\psi}^{2}} \boldsymbol{\beta}_{\mathrm{s}}^{2} \iint J^{2}\left(e_{2}^{2}+e_{3}^{2}\right) \sin \theta d \theta d \phi+1, \\
\frac{\left\langle T^{\prime 2}\right\rangle}{\mathcal{T}_{T}^{2}}= & \xi^{2(\gamma-1)},
\end{aligned}
$$

where $\boldsymbol{e}=(\cos \theta, \sin \theta \cos \phi, \sin \theta \sin \phi)^{T}, J=\int_{-t_{\mathrm{C}}}^{0} \xi^{2}\left(e_{1}^{2} \xi^{2}+e_{2}^{2}+e_{3}^{2}\right)^{-1} d t^{\prime}$, and $\mathcal{T}_{u}, \mathcal{T}_{\boldsymbol{\psi}}$, and $\mathcal{T}_{T}$ correspond to the turbulence intensity and scalar fluctuation at the beginning of the compression phase, viz.,

$$
\mathcal{T}_{u}=u_{\mathrm{s}}^{\prime}\left(=u_{\mathrm{s}}^{* \prime} \frac{\tau^{*}}{l_{1}^{*}}\right), \quad \mathcal{T}_{\boldsymbol{\psi}}=\boldsymbol{\psi}_{\mathrm{s}}^{\prime}\left(=\sqrt{\psi_{\mathrm{s}}^{* \prime 2}}\right), \quad \mathcal{T}_{T}=T_{\mathrm{s}}^{\prime}\left(=\frac{T_{\mathrm{s}}^{* \prime}}{T^{*}}\right)
$$


Equations (13) shows that the amplification of the turbulence and scalar fluctuations during the RCM compression phase are directly dependent on the initial turbulence intensity $\mathcal{T}_{u}$, scalar fluctuation $\mathcal{T}_{\psi}$, initial mixture stratification $\boldsymbol{\beta}_{\mathrm{s}}$, and the compression ratio $\xi$. In this context it is interesting to point out that the evolution of $\left\langle\boldsymbol{u}^{\prime 2}\right\rangle$ and $\left\langle\boldsymbol{\psi}^{\prime 2}\right\rangle$ does not depend on the mean strain rate $\alpha$. The direct consequence of this observation for practical RCM-operations is that the turbulence evolution is unaffected by the piston-speed, as long as it obeys the RDT-constraint of Eq. (8).

The temporal evolution of the turbulence and scalar fluctuations is obtained by evaluating Eqs. (13) as function of the compression ratio $\xi(t)$, which is obtained from the solution of Eqs. (3) and (7). The solution of these equations at $t=0$, corresponding to the end of the compression phase, provides information about the turbulence for the subsequent combustion phase, and the mathematical model is discussed in the next section.

\section{Ignition and Combustion Phase}

Following the compression phase, it is assumed that the combustion of the test gas mixture occurs through the autoignition mechanism. Specifically, homogeneously distributed ignition kernels in the adiabatic core region develop, leading to exothermic heat release and species conversion. The evolution of these ignition kernels is described through a stochastic formulation. To this end, a Lagrangian Fokker-Planck (LFP) model is developed which accounts for the turbulence interaction and scalar mixing between individual ignition kernels.

In the absence of a mean flow, the decay of the homogeneous turbulence during the combustion phase is described by the evolution equations for the turbulence kinetic energy $\langle k\rangle=\frac{1}{2}\left\langle u_{i}^{\prime 2}\right\rangle$ and dissipation rate $\langle\varepsilon\rangle$ as

$$
\begin{aligned}
& d_{t}\langle k\rangle=-\langle\varepsilon\rangle, \\
& d_{t}\langle\varepsilon\rangle=-C_{\varepsilon, 2} \frac{\langle\varepsilon\rangle}{\tau_{\mathrm{t}}} \quad \text { with } \quad \tau_{\mathrm{t}}=\frac{\langle k\rangle}{\langle\varepsilon\rangle},
\end{aligned}
$$

where $C_{\varepsilon, 2}$ is a constant, and $\tau_{\mathrm{t}}$ is the eddy life-time. The solution to Eqs. (15) can be described by a power-law decay as

$$
\langle k\rangle=\langle k\rangle_{0}\left(\frac{\tau_{0}}{t+\tau_{0}}\right)^{n}, \quad\langle\varepsilon\rangle=\langle\varepsilon\rangle_{0}\left(\frac{\tau_{0}}{t+\tau_{0}}\right)^{n+1},
$$

where the subscript " 0 " denotes the condition at $t=0$, corresponding to the end of the compression phase, and $\tau_{0}=n\langle k\rangle_{0} /\langle\varepsilon\rangle_{0}$. In this context it is pointed out that recent experimental investigations by Guibert et $a l .{ }^{13}$ support the fact that the turbulence during the combustion phase follows a geometric decay law, which can be adequately represented by Eqs. (16).

Using standard $k-\varepsilon$ closure modeling, $\tau_{0}$ and $\tau_{\mathrm{t}}$ can be expressed as

$$
\tau_{0}=\frac{n}{C_{D}} l_{m}\langle k\rangle_{0}^{-1 / 2}, \quad \tau_{\mathrm{t}}=\frac{1}{n}\left(t+\tau_{0}\right),
$$

where $\langle\varepsilon\rangle_{0}=C_{D}\langle k\rangle_{0}^{3 / 2} l_{m}^{-1}$ was used; ${ }^{16,18} n$ and $C_{D}$ are constants, and $l_{m}$ is a characteristic mixing length. These parameters are specified in Sec. III.

The evolution of the homogeneously distributed ignition kernels during the combustion phase are modeled using a Lagrangian Fokker-Planck equation, which can be written as ${ }^{19}$

$$
d\left(\begin{array}{c}
Z \\
C \\
T
\end{array}\right)=\left(\begin{array}{c}
0 \\
\dot{w}_{C} \\
\dot{w}_{T}
\end{array}\right) d t-\underline{\underline{A}}\left(\begin{array}{c}
Z-\langle Z\rangle \\
C-\langle C\rangle \\
T-\left\langle T_{\downarrow}\right\rangle
\end{array}\right) d t+\underline{\underline{B}} d \boldsymbol{W}(t),
$$

where $C$ is a reaction progress variable, which is here defined from a linear combination of major product mass fractions, $C=Y_{\mathrm{CO}_{2}}+Y_{\mathrm{CO}}+Y_{\mathrm{H}_{2} \mathrm{O}}+Y_{\mathrm{H}_{2}}$. The chemical source term for the progress variable is $\dot{w}_{C}$.

Heat-loss effects are incorporated in the LFP-model through the drift term (second term on the righthand-side) via the non-adiabatic mean temperature $\left\langle T_{\downarrow}\right\rangle$. This model follows the volume-expansion approach of Tanaka et al., ${ }^{20}$ and the value of $\left\langle T_{\downarrow}\right\rangle$ is determined from the solution of the time-dependent heat-equation, which is here written in cylindrical coordinates:

$$
\partial_{t} \Theta=(1-\Theta) d_{t} \ln \left(\langle T\rangle-T_{\mathrm{W}}\right)+\frac{1}{r} \partial_{r}\left(r \alpha \partial_{r} \Theta\right),
$$


where $\Theta=\left(T-T_{\mathrm{W}}\right) /\left(\langle T\rangle-T_{\mathrm{W}}\right), \alpha$ is the thermal diffusivity, and the boundary conditions for Eq. (19) are:

$$
\begin{array}{rll}
\left.\partial_{r} \Theta\right|_{r=0}=0 & \text { (Adiabatic core region) } \\
\Theta(r=R)=0 & \text { (Wall temperature). }
\end{array}
$$

From the time-dependent solution of Eq. (19), $\left\langle T_{\downarrow}\right\rangle$ can be computed as

$$
\left\langle T_{\downarrow}\right\rangle=\left(\langle T\rangle-T_{\mathrm{W}}\right) \frac{2}{R^{2}} \int_{0}^{R} \Theta r d r+T_{\mathrm{W}},
$$

and the thermal boundary layer thickness is

$$
\delta_{\downarrow}=\int_{0}^{R}(1-\Theta) d r .
$$

The chemical source term and heat-release rate are pre-evaluated from the homogeneous reactor calculations using a detailed chemical mechanism, and then tabulated in terms of $Z, C$, and $T$.

The vector $\boldsymbol{W}$ denotes independent Wiener processes, and a quantity in angular brackets, $\langle\phi\rangle$, is a volume-averaged mean value, which, for a spatially homogeneous flow, is identical to the mean value $\bar{\phi}$.

The drift term in Eq. (18) is modeled using the IEM model, for which the drift matrix $\underline{\underline{A}}$ is given by

$$
\underline{\underline{A}}=\left(\begin{array}{ccc}
\tau_{Z}^{-1} & 0 & 0 \\
0 & \left(\tau_{C}+\tau_{\downarrow}\right)^{-1} & 0 \\
0 & 0 & \tau_{T}^{-1}
\end{array}\right)=\left(\begin{array}{ccc}
\left\langle\chi_{Z}\right\rangle /\left\langle Z^{\prime 2}\right\rangle & 0 & 0 \\
0 & \frac{\left\langle\chi_{C}\right\rangle}{\left\langle C^{\prime 2}\right\rangle}\left(1+\tau_{\downarrow} \frac{\left\langle\chi_{C}\right\rangle}{\left\langle C^{\prime 2}\right\rangle}\right)^{-1} & 0 \\
0 & 0 & \left\langle\chi_{T}\right\rangle /\left\langle T^{\prime 2}\right\rangle
\end{array}\right) .
$$

where $\tau_{\downarrow}=\delta_{\downarrow}^{2} / \alpha$ is the thermal diffusion time scale. By introducing the time scale ratios ${ }^{21} C_{Z}=\tau_{\mathrm{t}} / \tau_{Z}$ and $C_{C}=\tau_{C} / \tau_{Z}$, and $\tau_{\mathrm{t}}$ from Eq. (17), $\underline{\underline{A}}$ can be written as

$$
\underline{\underline{A}}=\frac{C_{Z}}{C_{C} C_{T}} \frac{n}{\left(t+\tau_{0}\right)}\left(\begin{array}{ccc}
C_{C} C_{T} & 0 & 0 \\
0 & C_{T}\left(1+\frac{\delta_{\perp}^{2}}{\alpha} \frac{C_{Z}}{C_{C}} \frac{n}{t+\tau_{0}}\right)^{-1} & 0 \\
0 & 0 & C_{C}
\end{array}\right)
$$

where $C_{Z}$ and $C_{C}$ are constants of order unity and are given in Sec. III. The diffusion matrix $\underline{\underline{B}}$ enforces that the diffusion process is constrained to the accessible state-space, which can be represented as

$$
\underline{\underline{B}}^{2}=\underline{\underline{A}}\left(\begin{array}{c}
\left\langle Z^{\prime 2}\right\rangle f(Z) \\
\left\langle C^{2}\right\rangle f(C) \\
\left\langle T^{\prime 2}\right\rangle f(T)
\end{array}\right) \quad \text { with } \quad f(\psi)=\frac{\left(\psi-\psi^{-}\right)\left(\psi^{+}-\psi\right)}{\left\langle\left(\psi-\psi^{-}\right)\left(\psi^{+}-\psi\right)\right\rangle}
$$

where $\psi^{ \pm}$denotes the minimum and maximum value of the state-space variable.

In the following, Eq. (18) is solved for a large number of notional particles, representing individual ignition kernels. From this, mean field quantities for pressure and temperature are evaluated, and these results are compared with experimental data.

\section{Specification of Model Configuration}

The compression-ignition model, that was developed in the previous sections, is applied to the free-piston RCM at the University of Michigan. ${ }^{22}$ The UM-RCM facility consists of a pressurized driver section having a length of $5.54 \mathrm{~m}$ and an inner diameter of $154 \mathrm{~mm}$. The driven section, containing the test gas mixture, is separated from the driver section through a freely moving piston. The driven section is $2.54 \mathrm{~m}$ long and has an inner diameter of $101.2 \mathrm{~mm}$. The end of the driven section is connected to the test section with an inner diameter of $50.8 \mathrm{~mm}$ and a length of $50.6 \mathrm{~mm}$.

Since the present study is concerned with the adiabatic core region, the geometry of the UM-RCM facility can be simplified and represented by a cylinder of constant diameter. The dimensions are corrected to reproduce the geometric compression ratio of the facility. The length of the driven section, including the 
test section, is $l_{1}^{*}=2970 \mathrm{~mm}$, the length of the test section is $77 \mathrm{~mm}$, resulting in a compression ratio of $\xi=38.6$. The diameter of all sections is kept constant and equal to that of the UM-RCM with $101.2 \mathrm{~mm}$. The mass of the piston is $m^{*}=2.575 \mathrm{~kg}$, the pressure in the driver section is $p_{2}^{*}=2.0735$ bar, and friction is neglected. The initial pressure in the driven section is $p_{1}^{*}=0.09692 \mathrm{bar}$ and the ratio of specific heats is $\gamma=1.3$. Note that the cross-section area and the mass of the piston only affect the reference time scale $\tau^{*}$, which is evaluated to be $\tau^{*}=69.36 \mathrm{~ms}$.

The test gas in the driven section consists of a fuel-lean syngas/air mixture, and different syngas mixtures with the composition:

$$
\phi\left(a \mathrm{H}_{2}+\mathrm{CO}\right)+b \mathrm{CO}_{2}+\frac{1}{2}(1+a)\left(\mathrm{O}_{2}+c \mathrm{~N}_{2}\right)
$$

are considered. In this equation, $a, b$, and $c$ are the stoichiometric coefficients, and $\phi$ is the equivalence ratio, which can be expressed in terms of the mixture fraction through the relation ${ }^{21}$

$$
\phi=\frac{Z}{Z_{\mathrm{st}}} \frac{1-Z_{\mathrm{st}}}{1-Z},
$$

where $Z_{\text {st }}$ is the stoichiometric mixture fraction. The chemical mechanisms by Li et $_{\text {al }}{ }^{23}$ is used to describe the syngas combustion, and all model constants used in the LFP model are summarized in Tab. 1.

\begin{tabular}{cccccc}
\hline$n$ & $C_{D}$ & $C_{Z}$ & $C_{C}$ & $C_{T}$ & $l_{m}$ \\
\hline \hline 1.3 & 0.1664 & 2.0 & 1.0 & 1.0 & 0.0259 \\
\hline
\end{tabular}

Table 1. Model constants for RCM-model.

\section{Results}

\section{A. RCM Compression Phase}

The motion of the piston is obtained from Eq. (3), which is solved with the parameters specified in the previous section. Results for the piston location, piston velocity, and mean strain rate as function of time are illustrated in Fig. 2. Note that the time and all other quantities are non-dimensionalized, and $t=0$ corresponds to the end of the compression phase. From this figure, it is seen that the piston exhibits an approximately linear acceleration up to $t=-0.35$, after which the piston decelerates due to the compression of the test gas mixture in the driven section.

The mean strain rate, shown in Fig. 2(c), remains small up to $t=-0.35$. However, beyond this point $\alpha$ rapidly increases in magnitude until it reaches a value of -39.5 at the end of the compression phase.

\section{B. Effect of Compression Ratio on Turbulence Evolution}

The evolution of the turbulence and mixture fraction variance during the compression phase is shown in Fig. 3. These results are obtained by integrating the RDT-equations (13) using the compression ratio $\xi=x_{\mathrm{P}}^{-1}$ from the solution of Eq. (3). For this analysis the compression ratio is extended up to $\xi=100$, and the gray area illustrates the typical range of RCM compression ratios. The top panel in Fig. 3 shows the evolution of the normal stresses and the turbulence kinetic energy $k=\frac{1}{2}\left\langle u_{i}^{\prime 2}\right\rangle$. Note that the turbulence level in the driven section is only a function of the initial condition $\mathcal{T}_{u}$ and the current compression ratio, but - unlike the mixture fluctuations - is not affected by the temporal evolution of the compression phase. Following an initial transition, all normal stress components are linearly dependent on the compression ratio, with the $\left\langle u_{1}^{\prime 2}\right\rangle$-component growing fastest and exceeding the other components by a factor of two.

The results from this RDT analysis are particularly interesting, since they reveal the sensitivity of the turbulence level at the end of the RCM-compression phase on the initial conditions. In fact, Fig. 3 shows that for RCM-facilities, with compression ratios as high as 40, the initial turbulence is amplified by as a much as a factor of 50. This amplification is significant, and emphasizes the fact that care must be taken during the initialization and setup of RCM-experiments. The dependence of the mixture fraction variance on the compression ratio is shown in the bottom panel of Fig. 3. For these results the initial mixture fraction fluctuation and turbulence intensity are kept equal. 
(a) Piston Location

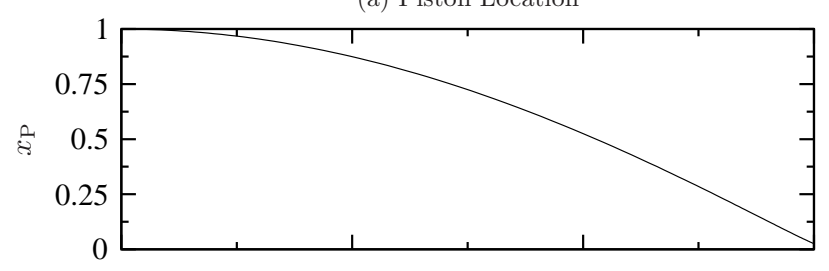

(b) Piston Velocity

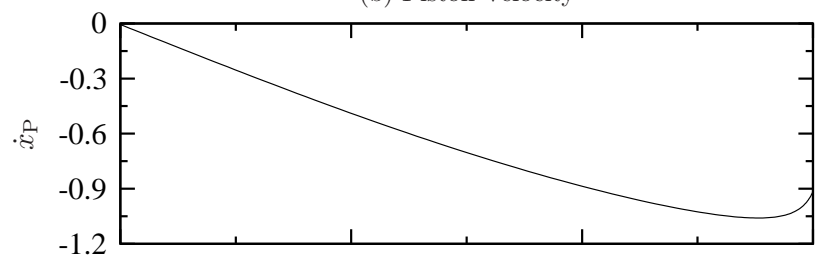

(c) Mean Strain Rate

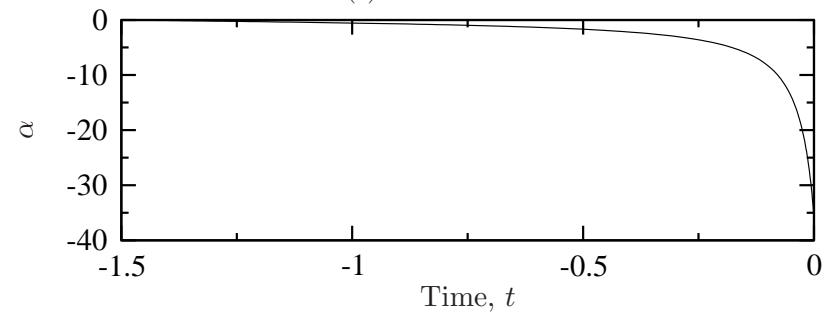

Figure 2. Results for the RCM-compression phase, obtained from the solution of Eq. (3), showing the temporal evolution of (a) the piston location $x_{\mathrm{P}},(\mathbf{b})$ the piston velocity $\dot{x}_{\mathrm{P}}$, and (c) the mean strain rate $\alpha=\dot{x}_{\mathrm{P}} / x_{\mathrm{P}}$.

Results for different values of mean mixture stratification are presented in Fig. 3(b). Since $\left\langle\boldsymbol{\psi}^{\prime 2}\right\rangle$ has a quadratic dependence on $\boldsymbol{\beta}_{\mathrm{s}}$, fluctuations of the mixture fraction are amplified due to the mean mixture stratification. This could become of importance when an internal mixture preparation is facilitated. In this context it is also noted, that the growth of $\left\langle\psi^{\prime 2}\right\rangle$ exhibits a dependence on the temporal evolution of the compression phase, which enters through the memory effect in the $J$-term in Eq. (13c): From the definition of $J$ it can be seen that for a given $\boldsymbol{\beta}_{\mathrm{s}}$ the mixture fluctuations can be reduced if shorter compression durations are used.

The amplification of temperature fluctuations as function of compression ratio is shown in the bottom graph of Fig. 3. From Eq. (13d) it can be seen $\left\langle T^{\prime 2}\right\rangle / \mathcal{T}_{T}^{2}$ follows the isentropic compression ratio, and amplifications by as much as factor of 5 to 10 can be observed for typical RCM-operating conditions.

\section{Effect of Initial Turbulence Level on the Ignition Dynamics}

In the following, we will study effects of initial turbulence and scalar fluctuations on the syngas combustion and ignition dynamics. The focus of this study is to assess effects of fluctuations of turbulence and mixture fraction. To this end, LFP-calculations are performed for different values of $\mathcal{T}_{u}$ and $\mathcal{T}_{Z}$, and 20000 particles $($ or ignition kernels) are used to obtain statistical quantities. The following mixture composition is considered: ${ }^{5}$ $\mathrm{H}_{2} / \mathrm{CO} / \mathrm{CO}_{2} / \mathrm{O}_{2} / \mathrm{N}_{2}=6.7 / 4.5 / 12.2 / 18.7 / 57.9$ (by volume) and $\phi=0.3$. The temperature and pressure at the end of the compression phase are $\left\langle T_{1}^{*}\right\rangle=944 \mathrm{~K}$ and $\left\langle p_{1}^{*}\right\rangle=11.2 \mathrm{bar}$, respectively. In the following, we will only consider adiabatic ignition problems, and effects of wall-heat losses on the ignition delay are addressed in Sec. D.

For the present case, the mean mixture stratification is set to zero, and $\mathcal{T}_{Z}$ is kept equal to $\mathcal{T}_{u}$. Note that $\mathcal{T}_{u}$ enters Eq. (18) through the time-scale $\tau_{0}$, which is dependent on $\langle k\rangle$ and $\mathcal{T}_{u}$ (see Eq. (17)). The composition of the particles is initialized from a beta-distribution: ${ }^{21}$

$$
P\left(Z ;\langle Z\rangle, \mathcal{T}_{Z}^{2}\right)=\frac{\Gamma(\alpha+\beta)}{\Gamma(\alpha) \Gamma(\beta)} Z^{\alpha-1}(1-Z)^{\beta-1}
$$


(a) Turbulence Fluctuations

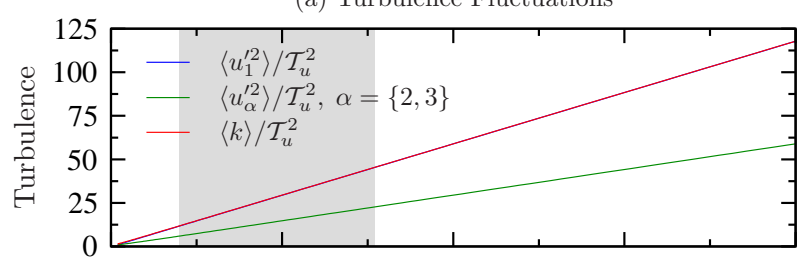

(b) Scalar Fluctuations

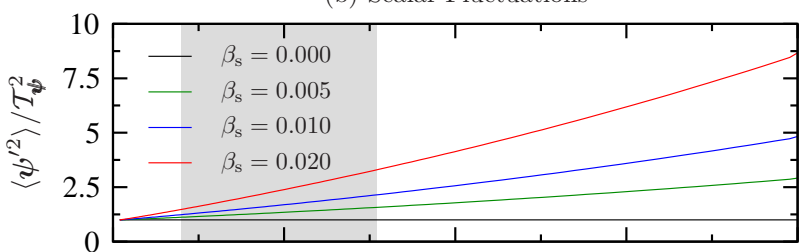

(c) Temperature Fluctuations

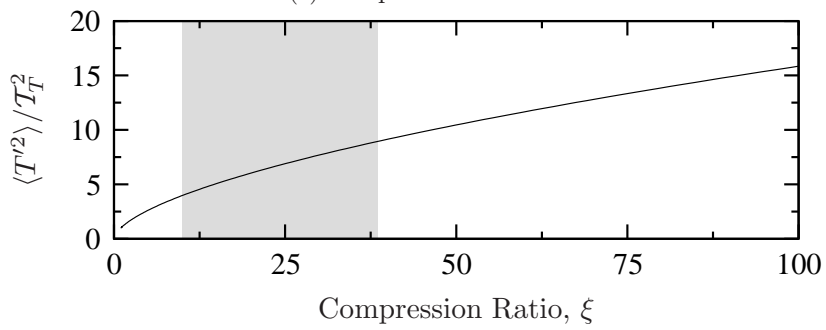

Figure 3. Dependence of turbulence and mixture fluctuations on the compression ratio, showing (top) turbulence normal stresses and turbulence kinetic energy, (middle) amplification of the scalar variance for different values of initial mean mixture stratification $\left(\mathcal{T}_{u}=\mathcal{T}_{\boldsymbol{\psi}}\right.$ is used to normalize $\left.\left\langle\psi^{\prime 2}\right\rangle\right)$, and (bottom) amplification of the temperature fluctuations. The turbulence kinetic energy is $\langle k\rangle=\frac{1}{2}\left\langle u_{i}^{\prime 2}\right\rangle$. The gray region illustrates the typical range of $\mathrm{RCM}$ compression ratios.

in which the coefficients $\alpha, \beta$, and $\gamma$ are expressed in terms of $\langle Z\rangle$ and $\mathcal{T}_{Z}^{2}$ :

$$
\alpha=\langle Z\rangle \gamma, \quad \beta=(1-\langle Z\rangle) \gamma, \quad \gamma=\frac{\langle Z\rangle(1-\langle Z\rangle)}{\mathcal{T}_{Z}^{2}}-1 .
$$

The progress variable for each particle is determined from the mixture composition for $Z$ and the condition at the end of the compression phase. Results for the temporal evolution of mean pressure and temperature are illustrated in Fig. 4. It can be seen that the LFP model agrees with the homogeneous reactor results (open symbols) for extremely small values of initial turbulence and scalar fluctuations, but deviates with increasing values of $\mathcal{T}_{u}$ and $\mathcal{T}_{Z}$. Higher turbulence levels lead to a reduction in the ignition delay. For this particular mixture composition the reduction in ignition delay can reach an order of magnitude for $\mathcal{T}_{u}=10^{-3}$. In this context it is noted that this value of $\mathcal{T}_{u}$ corresponds to a very modest root-mean-square (rms) velocity fluctuation of $4.3 \mathrm{~cm} / \mathrm{s}$, which is still two orders of magnitude smaller compared to equivalent flow regimes in internal combustion engines. ${ }^{24}$

From these results it is also evident that the turbulence not only affects the onset of the ignition but also the progress of reaction. Compared to the homogeneous reactor results, the slope of pressure and temperature decrease considerably. This behavior is also observed in the experiments. To substantiate this, an experimentally measured pressure trace $\mathrm{e}^{5,25}$ for the same operating conditions is shown in Fig. 4 . The modeling results show a qualitatively similar trend for the ignition behavior. Physically, this can be explained by the nonuniform behavior of individual ignition kernels, in which only a portion of the particles ignite, while the ignition process is delayed for other particles.

To complete this ignition analysis, we will assess the role of inhomogeneities in the species composition on the ignition behavior. To this end, $\mathcal{T}_{Z}$ is set to zero, so that all particles are initialized with the same mixture fraction. To account for inhomogeneities in the species composition, small perturbations in $C$ are added to the progress variable. These fluctuations are sampled from a homogeneous distribution, and its rms-value is denoted by $\mathcal{T}_{C}$. 

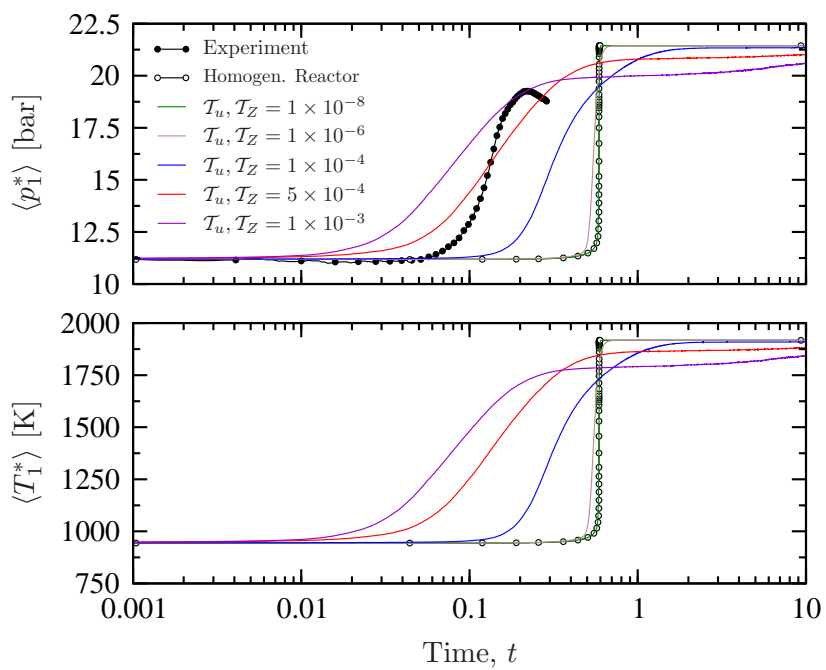

Figure 4. Evolution of mean pressure (top) and temperature (bottom) for different values of initial turbulence and scalar fluctuations. Open symbols correspond to homogeneous reactor results and the experimentally recorded pressure-history is shown by the closed symbols. ${ }^{5}$ The mixture composition is: $\mathrm{H}_{2} / \mathrm{CO} / \mathrm{CO}_{2} / \mathrm{O}_{2} / \mathrm{N}_{2}$ $=6.7 / 4.5 / 12.2 / 18.7 / 57.9$ (by volume) and $\phi=0.3$; temperature and pressure at the end of the compression phase are $\left\langle T_{1}^{*}\right\rangle=944 \mathbf{K}$ and $\left\langle p_{1}^{*}\right\rangle=11.2$ bar, respectively.

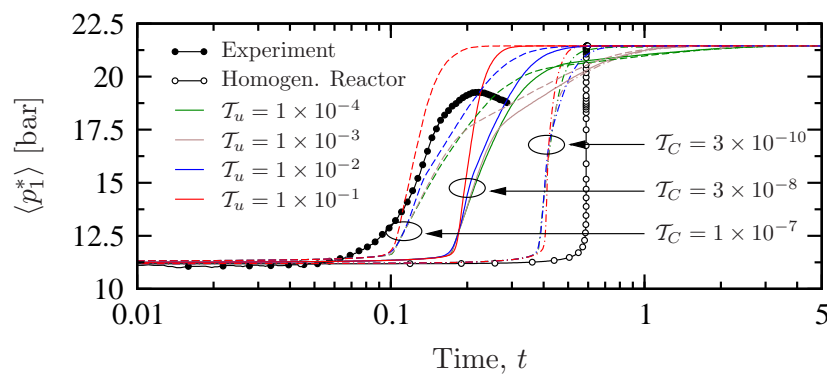

Figure 5. Evolution of mean pressure for different values of initial turbulence (indicated in the legend) and three different values of $\mathcal{T}_{C}$; Dash-dotted lines: $\mathcal{T}_{C}=3 \times 10^{-10}$; solid lines: $\mathcal{T}_{C}=3 \times 10^{-8}$; and dashed lines: $\mathcal{T}_{C}=1 \times 10^{-7}$. The mixture composition is: $\mathrm{H}_{2} / \mathrm{CO} / \mathrm{CO}_{2} / \mathrm{O}_{2} / \mathrm{N}_{2}=6.7 / 4.5 / 12.2 / 18.7 / 57.9$ (by volume) and $\phi=0.3$; temperature and pressure at the end of the compression phase are $\left\langle T_{1}^{*}\right\rangle=944 \mathrm{~K}$ and $\left\langle p_{1}^{*}\right\rangle=11.2$ bar, respectively.

Results obtained from a parametric study are presented in Fig. 5, showing the computed pressure traces for different values of $\mathcal{T}_{u}$. Results shown by the dash-dotted lines are obtained for $\mathcal{T}_{C}=3 \times 10^{-10}$, solid lines for $\mathcal{T}_{C}=3 \times 10^{-8}$, and the dashed lines correspond to $\mathcal{T}_{C}=1 \times 10^{-7}$. From this figure the following observations can be made. First, it is seen that the onset of ignition is solely controlled by the progress variable fluctuations, and turbulence plays only a secondary role in determining the ignition point. This observation is noteworthy as it is of direct relevance for practical applications. For instance, RCMs employing external mixture preparation establish very homogeneous compositions and negligible variations in equivalence ratio. However, wall heat transfer, mixture impurities, or surface-catalytic effects, as pointed out by Dryer \& Chaos, ${ }^{8}$ can induce small variations in temperature and species composition. Such effects can lead to exiguous perturbations in temperature and species composition, which are here represented by progress variable fluctuations. The second observation relates to the slope of the pressure rise. From this parametric study it is evident that with increasing turbulence intensity the reaction progress is delayed. In fact, it appears that for the cases with $\mathcal{T}_{u}=1 \times 10^{-3}$ combustion proceeds as a two-stage process which is reflected by a reduced pressure rise after about $60 \%$ of the maximum pressure is reached. The reason for this is the enhanced turbulent mixing leading to increased heat transfer and radical diffusion away from the ignition kernels. This, in turn, leads to extinction and reignition, which is reflected by a retarded pressure and temperature rise. 


\section{Wall Heat Loss Effects}

Non-adiabatic effects on the ignition and combustion process are discussed in this section. To this end, LFP-simulations are performed in which wall heat losses are considered by setting the wall temperature $T_{\mathrm{W}}$ to $850 \mathrm{~K}$. This value corresponds to $90 \%$ of the mixture temperature at the end of the compression phase. Simulation results for three different configurations are shown in Fig. 6. For all cases, the initial turbulence level is kept constant and identical to $\mathcal{T}_{u}=10^{-2}$, the initial stratification and fluctuations in mixture fraction and progress variable are set to zero, and three different levels of temperature fluctuations are considered. The mixture composition is identical to that considered in the previous section: $\mathrm{H}_{2} / \mathrm{CO} / \mathrm{CO}_{2} / \mathrm{O}_{2} / \mathrm{N}_{2}=$ $6.7 / 4.5 / 12.2 / 18.7 / 57.9$ (by volume) and $\phi=0.3$.
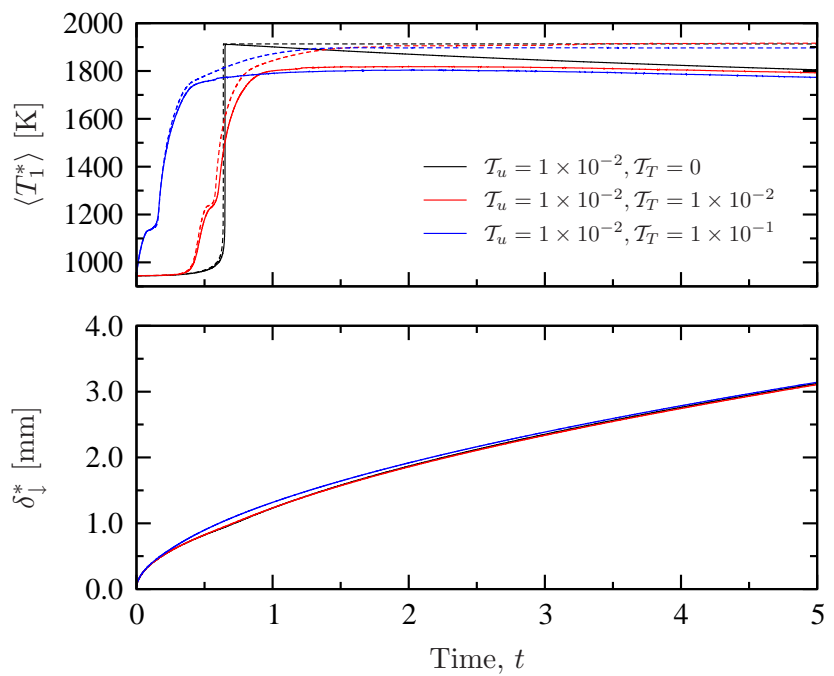

Figure 6. Comparison of temperature profile under consideration of wall heat loss effects (top) and temporal evolution of the thermal boundary layer thickness (bottom). Dashed lines correspond to adiabatic results, and solid lines denote results in which wall-heat loss effects are considered. The mixture composition is: $\mathrm{H}_{2} / \mathrm{CO} / \mathrm{CO}_{2} / \mathrm{O}_{2} / \mathrm{N}_{2}=6.7 / 4.5 / 12.2 / 18.7 / 57.9$ (by volume) and $\phi=0.3$; temperature and pressure at the end of the compression phase are $\left\langle T_{1}^{*}\right\rangle=944 \mathrm{~K}$ and $\left\langle p_{1}^{*}\right\rangle=11.2$ bar, respectively.

Results for the temperature evolution are shown in the top graph of Fig. 6. Non-adiabatic results are shown by the solid lines, and the dashed lines correspond to adiabatic simulations. This comparison shows that wall heat losses have only little effect on the ignition delay. This is partially attributed to the short ignition delay and the high temperature and pressure conditions. Only after the ignition, effects of wall heat losses are evident, leading to significant reduction in the temperature of the product mixture. It can also be seen that with increasing turbulence levels, the peak temperature is reduced, which is due to the competition between heat-generation and heat-losses to the wall.

The temporal evolution of the thermal boundary layer is illustrated in the bottom. It can be seen that the initial growth rate is well-approximated by $\delta_{\downarrow}^{*}=\sqrt{\alpha^{*} t^{*}}$; however, after ignition is achieved, a nearly linear growth-rate is observed, which can be attributed to the temperature-dependent increase in the thermal diffusivity.

\section{E. Syngas Ignition and Combustion}

The RCM ignition analysis is extended to a different syngas mixture and effects of initial turbulence and equivalence ratio fluctuations on the ignition delay over a temperature range of $600 \mathrm{~K} \leq\left\langle\mathrm{T}_{1}^{*}\right\rangle \leq 1300 \mathrm{~K}$ are investigated. For this investigation the syngas/air mixture composition is: $\mathrm{H}_{2} / \mathrm{CO} / \mathrm{CO}_{2} / \mathrm{O}_{2} / \mathrm{N}_{2}=$ $7.33 / 9.71 / 1.98 / 17.01 / 63.97$ and $\phi=0.5$, and the pressure at the end of the compression phase is $\left\langle p_{1}^{*}\right\rangle=20$ atm. The mechanism by Li et al. ${ }^{23}$ is used to describe the reaction chemistry.

Modeling results and comparisons with experimental data for ignition delay times are illustrated in Fig. 7. Results from the RCM model (lines) shows that effects of turbulence are mainly evident at low temperatures. However, with increasing levels of turbulence and mixture fluctuations, ignition delay times at higher temperatures become increasingly affected. This can be explained through a Damköhler number 


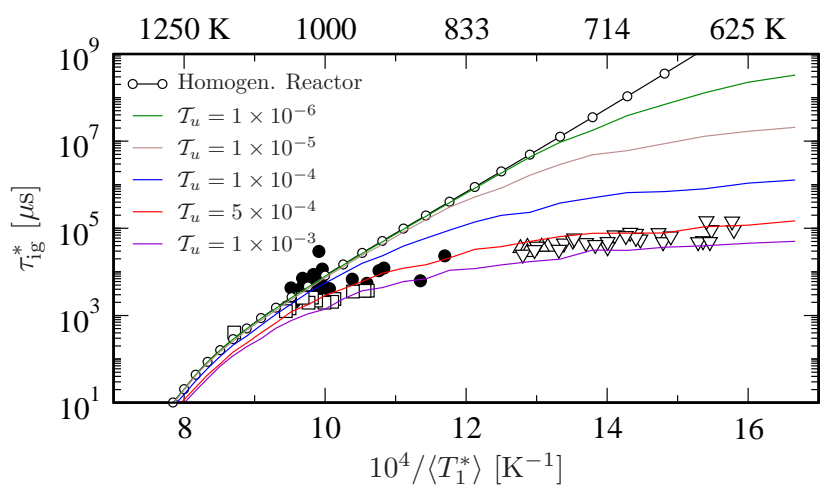

Figure 7. Ignition delay time of syngas/air mixtures. Experimental data are normalized to 20 atm assuming $1 / p$ proportionality. ${ }^{6}$ Symbols correspond to: RCM-measurements by Walton et al. ${ }^{\mathbf{5}} \bullet$; Shock tube measurements, $\square$, and flow reactor measurements, $\Delta$, by Petersen et al.; ${ }^{6}$ UTRC-flow reactor measurements, ${ }^{26} \nabla$. Simulation results for the syngas/air composition $\mathrm{H}_{2} / \mathrm{CO} / \mathrm{CO}_{2} / \mathrm{O}_{2} / \mathrm{N}_{2}=7.33 / 9.71 / 1.98 / 17.01 / 63.97$ (by volume) and $\phi=0.5$ are shown by lines. The initial pressure for all calculations is $\left\langle p_{1}^{*}\right\rangle=20 \mathrm{~atm}$, and the temperature increment is $25 \mathrm{~K}$. For the simulation, the initial value for the mixture fraction fluctuation is set equal to the initial turbulence level.

analysis, comparing the characteristic turbulence time scale with the ignition delay time:

$$
\mathrm{Da}_{\mathrm{ig}}=\frac{\tau_{0}^{*}}{\tau_{\mathrm{ig}}^{*}} \propto \frac{1}{\xi^{3 / 2}} \frac{1}{\mathcal{T}_{u}} \frac{\tau^{*}}{\tau_{\mathrm{ig}}^{*}}
$$

Large values of $\mathrm{Da}_{\mathrm{ig}}$ identify ignition-dominated processes which are unaffected by turbulent mixing. The Damköhler number is evaluated from the simulation results, and is illustrated in Fig. 8. Isocontours for

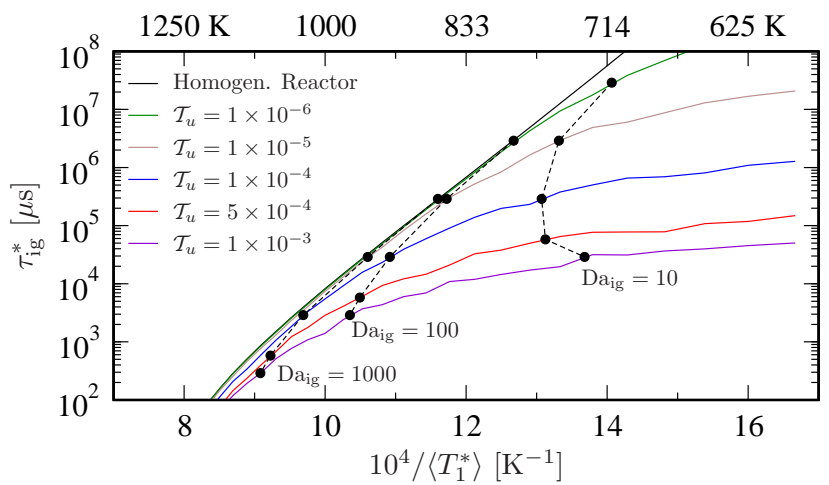

Figure 8. Damköhler number analysis for syngas ignition. Mixture composition and operating conditions are the same as in Fig. 7. The dashed lines with symbols correspond to curves of constant Damköhler numbers, $\mathrm{Da}_{\mathrm{ig}}=\{10,100,1000\}$.

three values of $\mathrm{Da}_{\mathrm{ig}}$ are shown. It can be seen that all ignition delay curves which are computed for different turbulence levels converge for $\mathrm{Da}_{\mathrm{ig}} \geq 100$, and asymptote to the homogeneous reactor results. This analysis suggests that Eq. (30) can be utilized as criterion to assess the significance of turbulent mixing and small-scale mixture fluctuations on the ignition process.

The RCM model accurately captures the experimentally observed trend that the ignition delay time asymptotes to constant value around $100 \mathrm{~ms}$ for low mixture temperatures (see Fig. 7). Although the data for $\left\langle T_{1}^{*}\right\rangle<800 \mathrm{~K}$ are obtained from flow reactor experiments, the RCM model predicts a similar behavior. This suggests that turbulence effects could also be of relevance in flow reactors, and further research is required to confirm this hypothesis. The best overall agreement between model results and experiments is obtained for $\mathcal{T}_{u}=\mathcal{T}_{Z}=5 \times 10^{-4}$ (red lines). For reference these values correspond to a rms-velocity of 2.1 $\mathrm{cm} / \mathrm{s}$ and mixture fraction fluctuation of $Z^{\prime}=\sqrt{\left\langle Z^{\prime 2}\right\rangle}=5 \times 10^{-4}$. Such magnitudes are reasonable, and in fact belong to the lower turbulence regime for technical flows. 


\section{Conclusions}

The role of turbulence on the syngas ignition in rapid compression machines over a wide range of operating conditions was studied. For this, a model was developed that describes the amplification of small-scale turbulence and mixture fluctuations during the RCM compression phase and the subsequent ignition process. In this model, it was assumed that these perturbations are generated during the filling process of the RCM with the fresh test gas mixture. Rapid distortion theory is used to characterize the turbulence amplification during the compression phase. The ignition process is described by a Lagrangian Fokker-Planck model, which considers the turbulent mixing between individual ignition kernels and fully accounts for detailed reaction chemistry of the lean syngas/air mixture. The model reduces to the well-known homogeneous reactor system in the absence of inhomogeneities in the initial flow field and mixture composition. With this model, important RCM-operating parameters could be identified, that are particularly relevant in controlling the ignition dynamics, namely initial levels of turbulence, scalar fluctuations, and temperature perturbations, mean mixture stratification, and the compression ratio.

Comparisons with experimental data showed that the model captures observed trends of retarded reaction progress and reduced ignition delay for increasing levels of initial turbulence and mixture fluctuations. Parametric studies were conducted for different initial turbulence and mixture conditions over a wide range of mixture temperatures. A Damköhler criterion was derived to assess the sensitivity of the induction chemistry to turbulence and mixture fluctuations in RCMs. Mixtures with Damköhler numbers below approximately 100 exhibit increasing sensitivities to turbulence fluctuations which is reflected by significant reduction in ignition delay times. Effects of reaction mechanisms on the computed ignition delay were found to be marginal and mostly confined to large Damköhler number conditions. The results show that the syngas ignition exhibits a pronounced sensitivity to the initial levels of turbulence and mixture fluctuations. Turbulence levels of less than $0.01 \%$ of the mean flow (corresponding to rms velocity fluctuations of a few $\mathrm{cm} / \mathrm{s}$ ) are sufficient in reducing the ignition delay time by several orders of magnitude.

Results of this study show that the turbulence/chemistry interaction plays an equally important role in affecting the syngas induction chemistry, and requires consideration in addition to chemical-kinetics and hydrodynamic process previously identified as leading mechanisms for the observed discrepancy under lowtemperature operating conditions.

\section{Acknowledgments}

This work was supported through the Office of Naval Research under contract N00014-10-1-0561 with Dr. H. Scott Coombe as technical monitor, and the Rackham Faculty Research Grant. We would like to express our gratitude to Margaret Wooldridge and Darshan Karwat for sharing their experimental data.

\section{References}

\footnotetext{
${ }^{1}$ International Energy Outlook. Report No. DOE/EIA-0484(2009), 2009 . $\quad$ available from http://www.eia.doe.gov/oiaf/ieo/index.html.

${ }^{2}$ K. H. Casleton, R. W. Breault, and G. A. Richards. System issues and tradeoffs associated with syngas production and combustion. Combust. Sci. Tech., 180:1013-1052, 2008.

${ }^{3}$ M. Chaos and F. L. Dryer. Syngas combustion kinetics and applications. Combust. Sci. Tech., 180:1053-1096, 2008.

${ }^{4}$ C.-J. Sung and C. K. Law. Fundamental combustion properties of $\mathrm{H}_{2} / \mathrm{CO}$ mixtures: Ignition and flame propagation at elevated pressures. Combust. Sci. Tech., 180:1097-1116, 2008.

${ }^{5}$ S. M. Walton, X. He, B. T. Zigler, and M. S. Wooldridge. An experimental investigation of the ignition properties of hydrogen and carbon monoxide mixtures for syngas turbine applications. Proc. Combust. Inst., 31:3147-3154, 2007.

${ }^{6}$ E. L. Petersen, D. M. Kalitan, A. B. Barrett, S. C. Reehal, J. D. Mertens, D. J. Beerer, R. L. Hack, and McDonell V. G. New syngas/air ignition data at lower temperature and elevated pressure and comparison to current kinetics models. Combust. Flame, 149:244-247, 2007.

${ }^{7}$ M. P. Burke, M. Chaos, F. L. Dryer, and Y. Ju. Negative pressure dependence of mass burning rates of $\mathrm{H}_{2} / \mathrm{CO} / \mathrm{O}_{2} / \mathrm{diluent}$ flames at low flame temperatures. Combust. Flame, 157:618-631, 2010.

${ }^{8}$ F. L. Dryer and M. Chaos. Ignition of syngas/air and hydrogen/air mixtures at low temperatures and high pressures: Experimental data interpretation and kinetic modeling implications. Combust. Flame, 152:293, 2008.

${ }^{9}$ J. F. Griffiths, Q. Jiao, W. Kordylewski, M. Schreiber, J. Meyer, and K. F. Knoche. Experimental and numerical studies of ditertiary butyl peroxide combustion at high pressures in a rapid compression machine. Combust. Flame, 93:303-315, 1993.

${ }^{10}$ D. Lee and S. Hochgreb. Rapid compression machines: Heat transfer and suppression of corner vortex. Combust. Flame, 114:531-545, 1998.
} 
${ }^{11}$ J. Würmel and J. M. Simmie. CFD studies of a twin-piston rapid compression machine. Combust. Flame, 141:417-430, 2005.

${ }^{12}$ G. Mittal and C.-J. Sung. Aerodynamics inside a rapid compression machine. Combust. Flame, 145:160-180, 2006.

${ }^{13}$ P. Guibert, A. Keromnes, and G. Legros. An experimental investigation of the turbulence effect on the combustion propagation in a rapid compression machine. Flow, Turb. Combust., 84(1):79-95, 2010.

14 J. C. R. Hunt. A review of the theory of rapidly distorted turbulent flows and its applications. Fluid Dyn. Trans., 9:121-152, 1977 .

${ }^{15}$ A. M. Savill. Recent developments in rapid-distortion theory. Ann. Rev. Fluid Mech., 19:531-575, 1987.

${ }^{16}$ P. A. Durbin and B. A. Pettersson Reif. Statistical Theory and Modeling for Turbulent Flows. John Wiley \& Sons, Inc., 2001.

${ }^{17}$ J. E. Elsworth, W. W. Haskell, and I. A. Read. Non-uniform ignition processes in rapid-compression machines. Combust. Flame, 13:437-438, 1969.

${ }^{18}$ S. B. Pope. Turbulent Flows. Cambridge University Press, Cambridge, 2000.

${ }^{19}$ R. O. Fox. Computational Models for Turbulent Reacting Flows. Cambridge University Press, Cambridge, 2003.

${ }^{20} \mathrm{~S}$. Tanaka, F. Ayala, and J. C. Keck. A reduced chemical kinetic model for HCCI combustion of primary reference fuels in a rapid compression machine. Combust. Flame, 133:467-481, 2003.

${ }^{21}$ N. Peters. Turbulent Combustion. Cambridge University Press, Cambridge, 2000.

${ }^{22}$ M. T. Donovan, X. He, B. T. Zigler, T. R. Palmer, M. S. Wooldridge, and A. Atreya. Demonstration of a free-piston rapid compression facility for the study of high temperature combustion phenomena. Combust. Flame, 137:351-365, 2004.

${ }^{23}$ J. Li, Z. Zhao, A. Kazakov, M. Chaos, F. L. Dryer, and J.J. Scire, Jr. A comprehensive kinetic mechanism for CO, $\mathrm{CH}_{2} \mathrm{O}$, and $\mathrm{CH}_{3} \mathrm{OH}$ combustion. Int. J. Chem. Kinet., 39:109-136, 2007.

${ }^{24}$ J. B. Heywood. Internal Combustion Engine Fundamentals. McGraw Hill, 1989.

${ }^{25}$ M. S. Wooldridge and D. Karwat, 2010. Private communication.

${ }^{26} \mathrm{~W}$. T. Peschke and L. J. Spadaccini. Determination of autoignition and flame speed characteristics of coal gases having medium heating value. Report No. EPRI-AP-4291, United Technologies Research Center, 1985. 